\title{
Review and Analysis of Crack Detection and Classification Techniques based on Crack Types
}

\author{
Sheerin Sitara. N. ${ }^{1}$, Kavitha. S. ${ }^{2}$, Raghuraman. G. ${ }^{2}$ \\ ${ }^{1}$ PG Scholar, Department of Computer Science and Engineering, SSN College of Engineering, Chennai, India. \\ ${ }^{2}$ Faculty of Computer Science and Engineering, SSN College of Engineering, Chennai, India.
}

\begin{abstract}
In real time scenario, cracks are very common in building, bridge, road, pavement, railway track, automobile, tunnel and aircraft. The presence of crack diminishes the value of the civil infrastructure and hence it is necessary to estimate the severity of crack. Crack detection and classification techniques with quantitative analysis play a major role in finding the severity of crack. The various quantitative metrics are length, width and area. Due to the rapid development in technology, number of images acquired for analysis is growing enormously. Therefore, automatic crack detection and classification techniques for civil infrastructure are essential. This paper focuses on three objectives: (i) Analysis of various crack detection and classification techniques based on crack types (ii) Implementation of Otsu's based thresholding method for crack detection (iii) Design of proposed system.
\end{abstract}

Keywords: Crack types, crack detection, crack classification, image processing, machine learning.

\section{INTRODUCTION}

Crack is a complete or incomplete separation of concrete into two or more parts, produced by breaking or fracturing. Different surfaces where crack can occur are buildings, bridges, roads, pavements, railway tracks, automobiles, tunnels, aircraft, etc. Cracks can be broadly classified into two categories namely active and dormant. In active cracks, the change in direction, width or depth occurs over a measured period of time whereas in dormant cracks it remains unchanged. If left unrectified, both active and dormant cracks provide passage for moisture penetration, which can lead to future damage. Some of the active cracks are longitudinal crack, transverse crack, miscellaneous crack, crocodile crack and reflection crack. Dormant cracks are very fine in nature and auto healing occurs over a time period. The various types of crack based on their structure are micro crack, thin crack, sealed crack, mixed crack, line-like crack, minor crack, tiny crack, medium crack, large crack and complex crack. In this paper, the research challenges and improvements in the field of crack detection and classification techniques are addressed.

Arun Mohan and Sumathi Poobal, 2016; have reviewed and analyzed different crack detection methods. In that paper, analysis is based on the objectives, image processing techniques, error level, accuracy level and datasets whereas, crack classification techniques and its issues are not addressed. In our paper, that issue has been addressed with analysis along with partial implementation. The paper is organized into three sections such as crack detection and crack classification techniques based on crack types, implementation of existing system and design of proposed system. In addition, the limitations of existing system and its solutions are discussed.

\section{A. Crack detection}

Crack detection is the method to identify the crack from the image automatically using image processing techniques. Different image processing techniques are segmentation, morphological operation, sobel edge detection method, cannel edge detection method, Otsu's method, gradient method, clustering method, least square method, histogram equalization method, particle filter, maximum entropy method, wiener filter and wavelet transform. The various image processing techniques used for crack detection is discussed in this section.

In railway track crack detection [Rizvi Aliza Raza et al., 2017], video camera is installed in separate sections of the track to acquire the images and the crack is detected using morphological operation, binarization, histogram equalization and de-noising. In addition to this, gabor Filters Invariant to Rotation method [Roberto medina et al., 2017] is used to exhibit different orientations along its length for single crack. This method is insensitive to brightness. Generally, plastic surface has line-like cracks and hence sequence of methods are applied to detect the cracks [Haiming Liu et al., 2016]. The methods are: reduction method to remove the noise, image gradient for reconstruction of the crack image, shape based optical model to identify the crack and circularity to find the shape. The proposed approaches are better than Otsu's method and clustering method. [Aliza et al., 2017], discussed that automobile or aircraft applications has minor crack and it can't be detected from single image and hence more time period is required. Image acquisition for a single case was done thrice such as $7 \mathrm{~m}$ above center, $7 \mathrm{~m}$ from right and $7 \mathrm{~m}$ from left. Then for crack detection, thresholding, morphological operation and canny edge detection are used. In [Mojtaba et al., 2016], preprocessing alleviates the adverse impact of non-uniform background and pavement markings, followed by morphological operation which enhances the posterior features to detect sealed crack in the pavement. 
Romulo et al., 2016, used color feature extraction method to differentiate outdoor images (sky, grass) from desirable features. This method classifies the segmented window based on color, particle filtering for selection of particles, clustering for crack detection and least square method for classification of crack type based on direction with quantitative analysis. Chen et al., 2016, identified the cracks from low resolution images and discontinuities in the image using wavelet transform and KD-tree. [Rabihamhaz et al., 2016] used Minimal Path Selection (MPS) method for crack detection and for evaluation Pseudo Ground Truth (PGT) and DSC (DICE Similarity Coefficient) rate are used. [Yuansen et al., 2016], discussed about thin crack in Reinforced Concrete (RC) bridges which requires lot of pen marking and hence it is difficult in real time applications. For crack detection, Stereo triangulation technique, least square method, optical flow analysis methods are applied.

In masonry wall [Ellenberg et al., 2014], discussed the damages that occurs around the surface roughness and patterns in masonry, edges from windows, doors and ends of the building which causes problems with edge detection of surface crack. In subway tunnel [Wenyu et al., 2014] crack detection, irrelevant object are differentiated from crack object. In concrete wall, dark crack is noticeably darker than the background and also unclear crack is brighter than ordinary cracks [Tomoyuki et al.,2008]. Improved percolation value (Alteration of threshold value) method detects the unclear crack in a manner similar to dark cracks. However, thresholding, segmentation and morphological operation are commonly used image processing techniques.

\section{B. Crack classification}

Crack classification is an approach to find the specific crack type using machine learning algorithms. Crack detection identifies or recognizes the presence of crack whereas crack classification classifies the crack based on the feature extracted from the crack region. Machine learning is a subfield of Artificial Intelligence (AI), useful to perform classification, prediction and clustering of the dataset depends on the application. Classification/Prediction is carried out using supervised learning algorithms whereas clustering is carried out using unsupervised algorithms. The different types of supervised learning algorithms applied for crack classification are Support Vector Machine (SVM), K Nearest Neighbors algorithm (KNN), Extreme Learning Machine (ELM), adaboost and random forest.

In underwater dam [Pengfeishi et al., 2017], it is difficult to detect and classify the cracks. Hence, solar images are used to detect and classify crack into tiny, medium and large using tensor voting method. [Salari and Ouyang, 2016] stated that images not only contains a road segment, but also includes other complicated background components. Crack in the pavement images with complicated background components such as trees, houses, etc. are also detected and classified using SVM, fractal thresholding and radon transform. Some of the images require preprocessing techniques for effective results.It includes wiener filter to remove the blurriness and reduction method to reduce the noise. [Chen et al., 2016] classifies the bridge crack into vertical, longitudinal, reflexive and crocodile cracks using SVM. Fuzzy clustering method [Nouha Ben et al., 2017] is useful when the input regions can't be defined clearly and precisely. As a combination of Fuzzy clustering method, k-means thresholding, segmentation, de-nosing, morphological operation and skeletonization gives an accuracy of $82 \%$ for pavement crack.

\section{RELATED WORK}

A detailed review based on crack type has been done for crack detection and classification. The first level crack types (minor, moderate and severe) and its appropriate subtypes are shown in Fig.1.

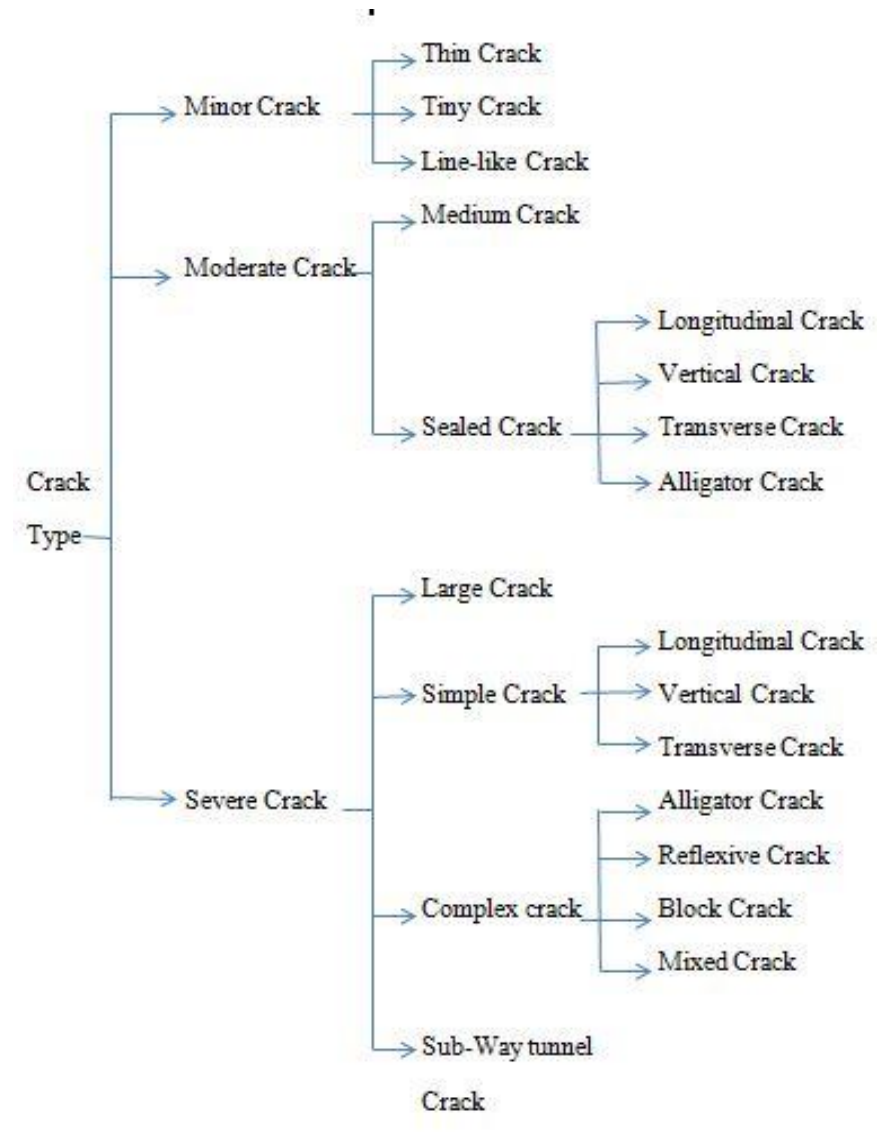

Figure 1. Crack type Classification

\section{A. Minor cracks}

Minor cracks are very small or thin crack with three sub types namely thin, tiny and line-like crack. This crack is common in RC bridges, underwater dam, plastic, automobiles and aircraft. Thin cracks [Yuansen et al., 2015] are common in RC bridges and can be detected using stereo triangulation technique, least square method and optical flow analysis method. These techniques are capable of capturing concrete surface cracks with width of 0.2 pixels using Region of Interest (ROI) and control point, whereas for better result the images should be captured using single camera without any change in the lighting effect. In underwater dam [Pengfeishi et al., 2017], it is difficult to detect and classify the cracks into tiny, medium 
and large crack and hence solar image is used. The methods applied in that paper are adaptive tensor voting, minimum spanning tree and K-means clustering. Line-like cracks [Haimingliu et al., 2016] are common in plastic surface and it follows sequence of methods to detect the cracks. The methods are: reduction method to remove the noise, image gradient for reconstruction of the crack image, shape based optical model to identify the crack and circularity to find the shape. The proposed approaches are better than Otsu's method and clustering method whereas discontinuities and quantitative analysis are not discussed. From analysis, it is noted that minor crack requires more time for crack detection and classification. Since cracks are small and has discontinuities, accuracy ranges between $80 \%$ and $86 \%$.

\section{B. Moderate cracks}

Moderate cracks are not much severe crack and hence remedial measures are required. In common, this crack occurs in underwater dam and concrete road with types such as medium, sealed and severe crack. Pengfeishi et al., 2017; stated that it is quite difficult to detect and classify the crack into tiny, medium and large crack and hence solar image is used. The methods applied in that paper are adaptive tensor voting, minimum spanning tree and K-means clustering. Sealed cracks [Mojtaba et al., 2016;] are common in concrete road with types such as longitudinal, vertical, transverse and alligator crack. It can be detected by using thresholding method, segmentation and morphological operation with high level of accuracy and consistency. The observed metric values of recall, precision, and accuracy are $87 \%, 98 \%$ and $93 \%$ respectively. From the analysis, it is inferred that the moderate crack sizes are larger than minor crack and hence discontinuities can be easily handled. The accuracy of moderate cracks classification ranges in between $93 \%$ and $93.3 \%$.

\section{Severe cracks}

Severe cracks are very large and dangerous crack and hence immediate remedial measures are required. This crack is very common in underwater dam, sub-way tunnel, bridges, pavements, concrete road and civil structure. Severe cracks include large, simple and complex cracks.

The large cracks [Pengfeishi et al., 2017] can be easily identified, but underwater dam cracks are difficult to detect and classify and hence solar image is used. The methods applied in this paper are adaptive tensor voting, minimum spanning tree and K-means clustering. This method is efficient than wasp colony algorithm and maintains an accuracy of $93.3 \%$ for large crack. [Wenyu Zhang et al., 2013] detect and classify sub-way tunnel crack using morphological operation, thresholding operation, ELM, radial basis function neural network (RBF), SVM, and KNN. For that, the accuracy rate is $90 \%$.

From analysis, it is observed that severe cracks can be classified even in the presence of complicated backgrounds like tree, houses, etc., and resulted with a accuracy of $90 \%$.
Accuracy level for severe cracks is around 95\%. The summary of severe cracks is given in Table 1 for better readability.

Table 1: Severe Crack

\begin{tabular}{|c|c|c|c|}
\hline $\begin{array}{c}\text { Crack } \\
\text { type }\end{array}$ & $\begin{array}{c}\text { Crack } \\
\text { surface }\end{array}$ & $\begin{array}{l}\text { Crack detection } \\
\text { techniques }\end{array}$ & $\begin{array}{c}\text { Crack } \\
\text { Classification } \\
\text { techniques }\end{array}$ \\
\hline $\begin{array}{l}\text { Large } \\
\text { crack }\end{array}$ & $\begin{array}{l}\text { Underwater } \\
\text { dam }\end{array}$ & $\begin{array}{l}\text { Particle filter } \\
\text { method }\end{array}$ & $\begin{array}{l}\text { K-means clustering, } \\
\text { adaptive tensor } \\
\text { voting and minimum } \\
\text { spanning tree }\end{array}$ \\
\hline $\begin{array}{l}\text { Sub- } \\
\text { way } \\
\text { tunnel } \\
\text { crack } \\
\end{array}$ & $\begin{array}{l}\text { Sub-way } \\
\text { tunnel }\end{array}$ & $\begin{array}{l}\text { Morphological } \\
\text { operation, } \\
\text { thresholding } \\
\text { operation }\end{array}$ & $\begin{array}{l}\text { RBF, SVM, and } \\
\text { KNN }\end{array}$ \\
\hline
\end{tabular}

\section{Simple cracks}

Simple cracks are less complex and it includes longitudinal, vertical and transverse crack. Longitudinal crack are common in bridges, pavements, concrete road and civil structure.

Table 2: Simple Cracks

\begin{tabular}{|l|l|l|l|}
\hline Crack type & \multicolumn{1}{|c|}{ Surface } & $\begin{array}{l}\text { Crack detection } \\
\text { techniques }\end{array}$ & $\begin{array}{c}\text { Crack } \\
\text { Classification } \\
\text { techniques }\end{array}$ \\
\hline $\begin{array}{l}\text { Longitudinal } \\
\text { crack }\end{array}$ & $\begin{array}{l}\text { Bridges, } \\
\text { pavements, } \\
\text { concrete } \\
\text { road and } \\
\text { civil } \\
\text { structure }\end{array}$ & $\begin{array}{l}\text { Wavelet transform, } \\
\text { morphological } \\
\text { operation, KD-tree, } \\
\text { EMD method, } \\
\text { binarization, region } \\
\text { growing method }\end{array}$ & $\begin{array}{l}\text { SVM, random } \\
\text { forest and } \\
\text { adaboost }\end{array}$ \\
\hline $\begin{array}{l}\text { Vertical } \\
\text { crack }\end{array}$ & $\begin{array}{l}\text { Bridges, } \\
\text { concrete } \\
\text { road and } \\
\text { civil } \\
\text { thresholding }\end{array}$ & $\begin{array}{l}\text { Particle filtering, } \\
\text { sobel edge } \\
\text { detection method, } \\
\text { least square } \\
\text { method, wavelet } \\
\text { transform, } \\
\text { morphological } \\
\text { operation, KD- tree }\end{array}$ & $\begin{array}{l}\text { SVM, random } \\
\text { forest and } \\
\text { adaboost }\end{array}$ \\
\hline $\begin{array}{l}\text { Diagonal } \\
\text { crack }\end{array}$ & $\begin{array}{l}\text { Concrete } \\
\text { road, } \\
\text { concrete } \\
\text { pavement } \\
\text { and civil } \\
\text { structure }\end{array}$ & $\begin{array}{l}\text { Thresholding } \\
\text { method, } \\
\text { segmentation, } \\
\text { morphological } \\
\text { operation, color } \\
\text { feature extraction } \\
\text { method, particle } \\
\text { filtering, sobel } \\
\text { edge detection } \\
\text { method, least } \\
\text { square method, } \\
\text { fractal } \\
\text { thresholding, radon } \\
\text { transform }\end{array}$ & $\begin{array}{l}\text { SVM, random } \\
\text { forest and } \\
\text { adaboost }\end{array}$ \\
\hline
\end{tabular}


It can be detected using wavelet transform, morphological operation, KD-tree, EMD method, binarization, region growing method and fractal thresholding and classified using SVM, random forest and adaboost[Romulo et al., 2016; Chen et al., 2016; Weili et al., 2017].Vertical crack are common in bridges, concrete road and civil structure and can be detected using particle filtering, sobel edge detection method, least square method, wavelet transform, morphological operation, KD- tree and classified using SVM, random forest and adaboost [Mojtaba et al., 2016; Romulo et al., 2016; Chen et al., 2016]. Transverse crack are common in concrete road, concrete pavement and civil structure and can be detected using thresholding method, segmentation, morphological operation, color feature extraction method, particle filtering, sobel edge detection method, least square method, fractal thresholding, radon transform and classified using SVM, random forest and adaboost [Mojtaba et al., 2016; Salari and Ouyang, 2016; Romulo et al., 2016]. Simple cracks from low resolution images and discontinuities in the image can also be identified using wavelet transform and $\mathrm{KD}$ - tree. The summary of simple cracks is given in Table 2 for better readability.

\section{Complex cracks}

Complex cracks are complex in shape and direction and hence more details are required for classification. Complex cracksare common in bridges, pavements, concrete road and civil structure. Complex cracks includes alligator, reflexive, block and mixed crack. Alligator crack are common in bridges, concrete pavement and can be detected using thresholding method, segmentation, morphological operation, EMD method, binarization, radon transform, region growing method, least square method and classified by SVM, random forest and adaboost [Mojtaba et al., 2016; Salari and Ouyang, 2016; Chen et al., 2016; Weili et al., 2017]. Reflexive crack are common in bridges and can be detected using wavelet transform, morphological operation, KD- tree and SVM [Chen et al., 2016]. Block crack are common in concrete pavement and can be detected using fractal thresholding, radon transform classified using SVM, random forest adaboost [Salari and Ouyang, 2016]. Mixed crack are common in asphalt pavement and can be detected using gabor filter method [Salman et al., 2013]. The summary of complex cracks is given in Table 3.

From analysis, some conclusions are derived. (i) particle filter algorithm is best suitable for underwater crack detection, (ii). In classification, random forest algorithm and adaboost resulted better accuracy than K-nearest neighbor, (iii) Otsu's method is widely used for crack detection. Otsu's based thresholding approach has been implemented to find the limitations of existing system for various image acquisition conditions.
Table 3: Complex Cracks

\begin{tabular}{|c|c|c|c|}
\hline $\begin{array}{c}\text { Crack } \\
\text { type }\end{array}$ & Surface & $\begin{array}{l}\text { Crack detection } \\
\text { techniques }\end{array}$ & $\begin{array}{c}\text { Crack } \\
\begin{array}{c}\text { Classification } \\
\text { techniques }\end{array} \\
\end{array}$ \\
\hline $\begin{array}{l}\text { Alligator } \\
\text { crack }\end{array}$ & $\begin{array}{l}\text { Bridges, } \\
\text { concrete } \\
\text { pavement }\end{array}$ & $\begin{array}{l}\text { Thresholding method, } \\
\text { segmentation, } \\
\text { morphological } \\
\text { operation, EMD } \\
\text { method, binarisation, } \\
\text { radon transform, region } \\
\text { growing method, Least } \\
\text { square method }\end{array}$ & $\begin{array}{l}\text { SVM, random } \\
\text { forest } \\
\text { andadaboost }\end{array}$ \\
\hline $\begin{array}{l}\text { Reflexive } \\
\text { crack }\end{array}$ & Bridges & $\begin{array}{l}\text { wavelet transform, } \\
\text { morphological } \\
\text { operation, KD- tree }\end{array}$ & SVM \\
\hline $\begin{array}{l}\text { Block } \\
\text { crack }\end{array}$ & $\begin{array}{l}\text { Concrete } \\
\text { pavement }\end{array}$ & $\begin{array}{l}\text { Fractal } \\
\text { thresholding,radon } \\
\text { transform }\end{array}$ & $\begin{array}{l}\text { SVM, random } \\
\text { forest } \\
\text { andadaboost }\end{array}$ \\
\hline $\begin{array}{l}\text { Mixed } \\
\text { crack }\end{array}$ & $\begin{array}{l}\text { Asphalt } \\
\text { pavement }\end{array}$ & Gabor filter method & \\
\hline
\end{tabular}

\section{IMPLEMENTAYTION OF EXISTING WORK}

Most of the existing system uses Otsu's thresholding based method because of its global automatic thresholding principle. Otsu's method is used to detect the crack because it is based on class-invariance principle i.e.; within class variance is minimum and between class variance is maximum.

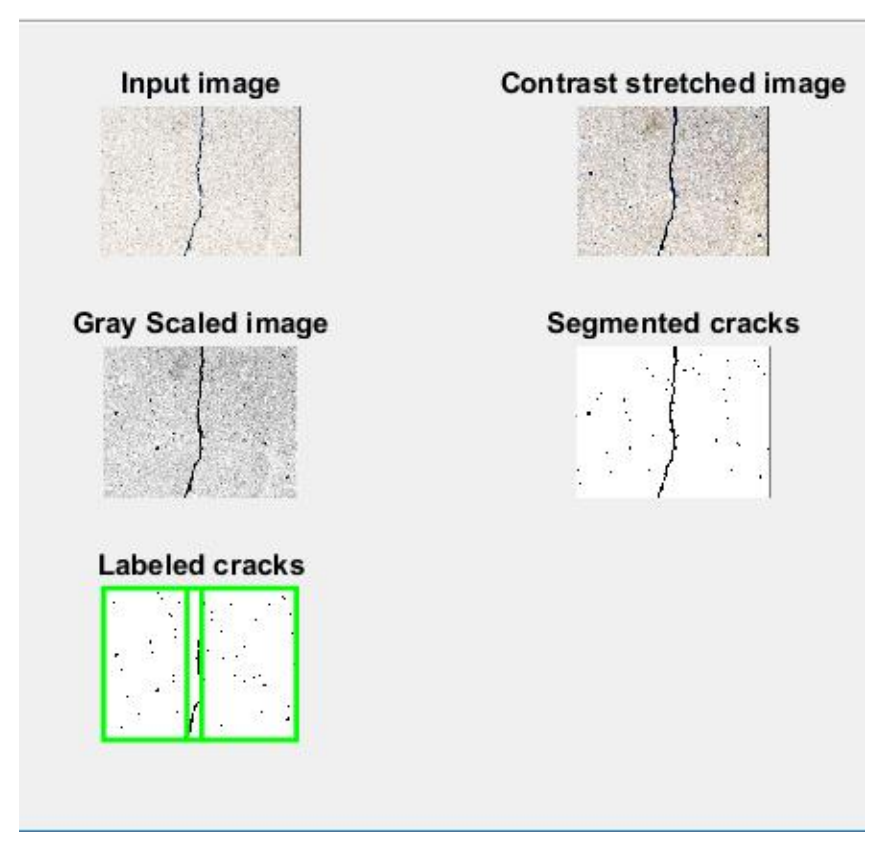

Figure 2.a) Preprocessing module b) Resize the image c) Normalizing the intensity value d) Segmentation by thresholding e) Extracting crack region (labeledcracks are highlighted in green color) 
In Fig.2, stage I represents input image. In stage II, the contrast stretched image is obtained through normalization of the intensity value. In stage III, contrast stretched image is converted into gray scaled image. In stage IV, by optimum threshold crack region are segmented from non-crack region. In stage $\mathrm{V}$, crack region is shown in green color. More sample set of output are shown in Fig.3 and Fig.4.

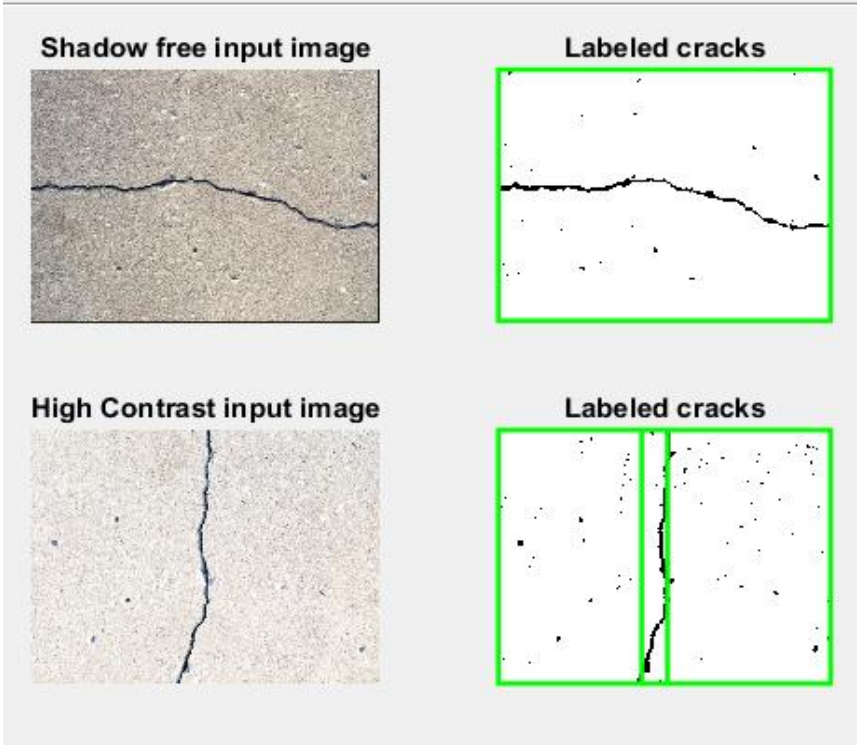

Figure 3.a) Shadow free image b) Result of shadow free image c) High contrast image d) Result of high contrast image

From the visualization of Fig.4, the detected crack region is not accurate for shadow and low contrast images. It also labels some of the non-crack region as crack region. Discontinuities in crack region are also not resolved.

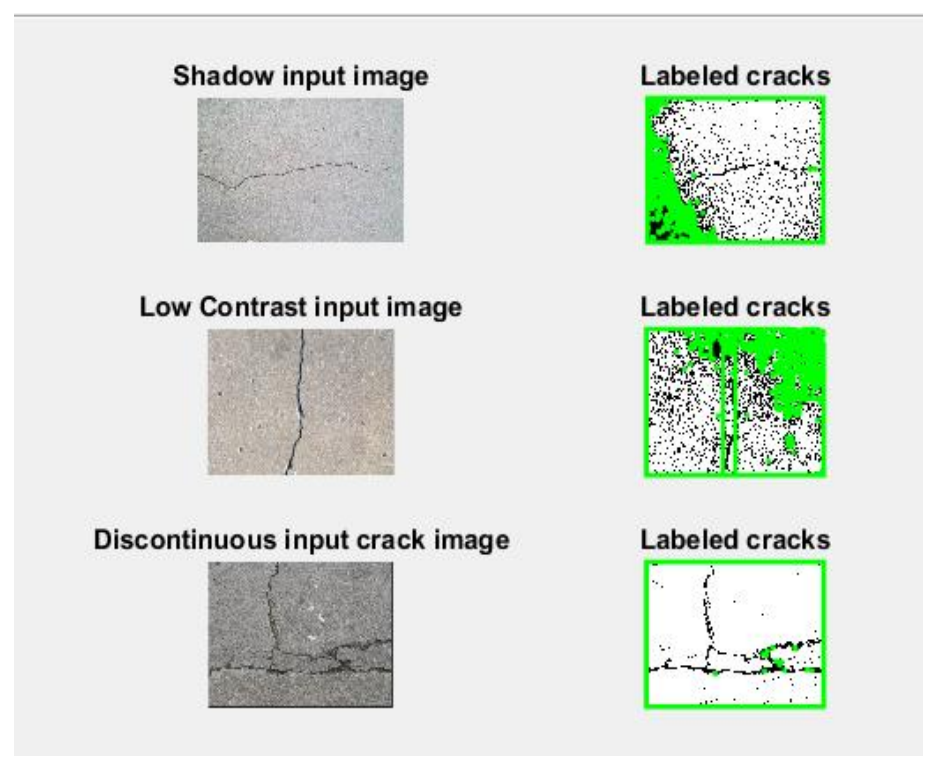

Figure.4.a) Shadow image b) Result of shadow image c) Low contrast image d) Result of low contrast image e) Discontinuities crack image f) Result of discontinuities crack image

\section{PROPOSED SYSTEM}

Automatic crack detection and classification system is proposed for concrete surface images. In pre-processing step, blurness of the image is removed, using Wiener filter. Cracks in poor contrast and non-uniform illumination images are not easy to identify and may yield erroneous results. To overcome these issues, Wavelet transform and Singular Value Decomposition (SVD) are proposed. Gray scale processing of images for existing crack detection methods are seems to be good enough, but unaffected areas are also identified as cracks. To address this issue, morphological operation and KD-tree is included in the design. Morphological operation is used to enhance the image for crack segmentation whereas $\mathrm{KD}$ tree is applied to connect the discontinuities in the crack region. For classification of cracks into specific type from the extracted features random forest algorithm is selected, since it resulted better accuracy in literature. Finally, quantitative analysis in terms of length, width, area and number of cracks are calculated. The design of the proposed system is shown in Fig.5.

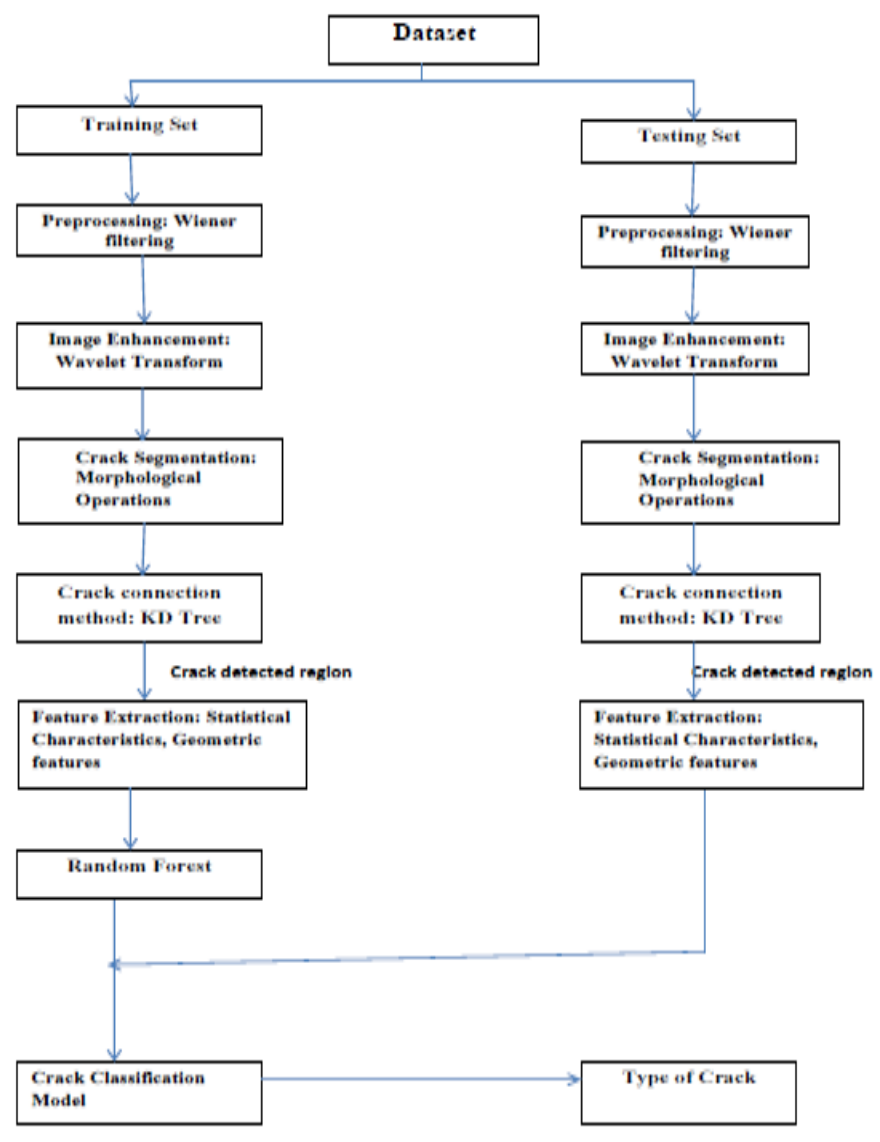

Figure 5. Image Enhancement using Wavelet transform and SVD

\section{CONCLUSION}

This paper presents the survey of different crack detection and classification techniques followed by implementation of existing Otsu's based method for crack detection. From this implementation, the limitations are inferred and a new design is proposed to address the limitations such as false crack 
detection for shadow or low contrast image and discontinuities in crack region. In the future, we plan to rectify these issues using wavelet transform and SVD with random forest algorithm for crack classification. Finally, the analysis is carried out on the basis of image processing and machine learning techniques. The results have to be analyzed using appropriate quantitative metrics of crack detection and classification.

\section{REFERENCES}

[1] Mojtaba Kamaliardakani., Lu Sun and Mostafa K. Ardakani., 2016, "Sealed-Crack Detection Algorithm using Heuristic Thresholding Approach", Journal of Computing in Civil Engineering., 30(1).

[2] Salari, E and Ouyang, D., 2016, “An Image-based Pavement Distress Detection and Classification”, IEEE International Conference on Electro/Information Technology"., pp. 1-6.

[3] Salman M., Mathavan S., Kamal K., Rahman M., 2013, "Pavement Crack Detection using the Gabor Filter", International IEEE Annual Conference on Intelligent Transportation Systems., pp. 2039 - 2044.

[4] Romulo Gonçalves Lins and Sidney N. Givigi., 2016, "Automatic Crack Detection and Measurement based on Image Analysis", IEEE Transactions on Instrumentation and Measurement., 65(3), pp.583 590.

[5] ChenYao., Mei Tao., Wang Xiaojie., LiFeng., 2016, "A Bridge Crack Image Detection and Classification Method based on Climbing Robot”, IEEE International Conference on Chinese Control Conference (CCC), pp. 4037 - 4042.

[6] Rabih Amhaz., Sylvie Chambon., Jerome Idier and Vincent Baltazart., 2016, "Automatic Crack Detection on Two-Dimensional Pavement Images: An Algorithm based on Minimal Path Selection", IEEE Transactions on Intelligent Transportation Systems 17(10), pp. $2718-2729$.

[7] Pengfei Shi., Xinnan Fan., Jianjun Ni., Zubair Khan., Min Li., 2017, “A Novel Underwater Dam Crack Detection and Classification approach based on Sonar Images”, PLOS ONE, 12(6), pp. 1-17

[8] Wei Li., Ju Huyan., Susan L. Tighe., Qing-qing Ren and Zhao-yun Sun., 2017, "Three-Dimensional Pavement Crack Detection Algorithm based on TwoDimensional Empirical Mode Decomposition", Journal of Transportation Engineering., 143(2).

[9] Wenyu Zhang., Zhenjiang Zhang., Dapeng Qi and Yun Liu., 2014, "Automatic Crack Detection and Classification Method for Subway Tunnel Safety Monitoring”, Sensors., ISSN. 1424 - 8220, pp. 19307-19328.

[10] Yuan-Sen Yang., Chung-Ming Yang., Chang-Wei Huang., 2015, "Thin Crack Observation in a
Reinforced Concrete Bridge Pier Test using Image Processing and Analysis", Advances in Engineering Software, Vol.83, pp.99-108.

[11] Nikhil Chaudhari., Akshat Sharma and Anisha M. Lal., 2017, "Crack Detection using Image Processing for Automobiles and Aircrafts”, International Journal of Engineering \& Technology for Automobile Security., 1(1), pp. 17-28.

[12] Hai-ming Liu., Zhang-can Huang., Yu-ting Zang and Ahmed Mahgoub Ahmed Talab., 2016, "Shape based Micro Crack Detection in Plastic through Image Processing”, International Journal of Signal Processing, Image Processing and Pattern Recognition., 9(1), pp.281-288.

[13] Aiguo Ouyang., Chagen Luo., and Chao Zhou., 2010, "Surface Distresses Detection of Pavement based on Digital Image Processing”, International Conference on Computer and Computing Technology in Agriculture., pp.368-375.

[14] Nouha Ben Cheikh Ahmed., Samer Lahouar., Chokri Souani., Kamel Besbes., 2017, “Automatic Crack Detection from Pavement Images using Fuzzy Thresholding”, International Conference on Control, Automation and Diagnosis., pp. 528 - 537.

[15] Prateek Prasanna., Kristin J. Dana, Nenad Gucunski., Basily B. Basily., Hung M. La., Ronny Salim Lim., and Hooman Parvardeh., 2016, "Automated Crack Detection on Concrete Bridges”, IEEE Transactions on Automation Science and Engineering, 13(2), pp. $591-599$.

[16] Roberto Medina., José Llamas., Jaime GómezGarcía-Bermejo., Eduardo Zalama and Miguel José Segarra., 2017, "Crack Detection in Concrete Tunnels using a Gabor Filter Invariant to Rotation”, Sensors., 17(7).

[17] Rizvi Aliza Raza, Khan Pervez Rauf., Ahmad Shafeeq., 2016, "Crack Detection in Railway Track using Image Processing”, International Journal of Advance Research, Ideas and Innovations in Technology., 3(4), pp. 489-496.

[18] Fábio Celestino Pereira, Carlos Eduardo Pereira., 2015, "Embedded image processing systems for automatic recognition of cracks using UAVs", IFAC., 48(10).

[19] Yong Shi., Limeng Cui., Zhiquan Qi., Fan Meng., and Zhensong Chen., 2016, "Automatic Road Crack Detection using Random Structured Forests”, IEEE Transactions on Intelligent Transportation Systems., 17(12), pp. $3434-3445$.

[20] Henrique Oliveira., and Paulo Lobato Correia., 2013, "Automatic Road Crack Detection and Characterization”, IEEE Transactions on Intelligent Transportation Systems., 14(1), pp.155 - 168. 
International Journal of Applied Engineering Research ISSN 0973-4562 Volume 13, Number 8 (2018) pp. 6056-6062

(C) Research India Publications. https://dx.doi.org/10.37622/IJAER/13.8.2018.6056-6062

[21] Hoang-Nam Nguyen., Tai-Yan Kam and Pi-Ying Cheng., 2012, "A Novel Automatic Concrete Surface Crack Identification using Isotropic Undecimated Wavelet Transform”, IEEE International Symposium on Intelligent Signal Processing and Communication Systems., pp. $766-771$.

[22] Henrique Oliveira., Paulo Lobato Correia., 2008, "Identifying and Retrieving Distress Images from Road Pavement Surveys”, IEEE ICIP., pp. 57 - 60.

[23] Meng Qiao., Wang Xiaoying., and Zhang Yu an., 2016, "Research on a Least Squares Thresholding Algorithm for Pavement Crack Detection”, Sixth International conference on Information Science and Technology., pp. $465-469$.

[24] Ganesh Nagasai Prasad, V., Habibullah khan., Bhavana, K., Muralidhar, Ch. Tulasisaikiran., 2012, "Image Enhancement using Wavelet transforms and $S V D$ ”, International Journal of Engineering Science and Technology (IJEST)., 5(7), pp. $594-599$.

[25] Arun Mohan and Sumathi Poobal., 2017, "Crack detection using image processing: A critical review and analysis", Alexandria Engineering Journal., DOI:10.1016/j.aej.2017.01.020, article in press. 